\title{
MicroRNA-567 dysregulation contributes to carcinogenesis of breast cancer, targeting tumor cell proliferation, and migration
}

\author{
Gloria Bertoli ${ }^{1}$ (I) Claudia Cava $^{1} \cdot$ Cecilia Diceglie $^{2,3} \cdot$ Cristina Martelli $^{3}$. \\ Giampiero Rizzo $^{4} \cdot$ Francesca Piccotti $^{4,5} \cdot$ Luisa Ottobrini $^{1,3} \cdot$ Isabella Castiglioni $^{1}$
}

Received: 5 July 2016/ Accepted: 8 December 2016/Published online: 20 December 2016

(c) The Author(s) 2016. This article is published with open access at Springerlink.com

\begin{abstract}
Purpose We demonstrated that Hsa-miR-567 expression is significantly downregulated in poor prognosis breast cancer, compared to better prognosis breast cancer, having a role in the control of cell proliferation and migration by regulating KPNA4 gene.

Methods and results In this study, based on our previously published in silico results, we proved both in vitro (cell line studies) and ex vivo (clinical studies), that Hsa-miR-567 expression is significantly downregulated in breast cancer with poor prognosis when compared to breast cancer with better prognosis. More intriguingly, we demonstrated that the ectopic expression of Hsa-miR-567 in poor prognosis breast cancer cell line strongly inhibits in vitro cell proliferation and migration. Furthermore, we showed in vivo that breast cancer cells, stably expressing Hsa-miR-567, xenografted in mouse, reduce tumor growth ability. Consistently, we found that karyopherin 4 (KPNA4), predicted target gene of Hsa-miR-567 as identified by our in silico
\end{abstract}

Gloria Bertoli

gloria.bertoli@ibfm.cnr.it

1 Institute of Molecular Bioimaging and Physiology, National Research Council (IBFM-CNR), Via F.Cervi 93,

20090 Segrate-Milan, Milan, Italy

2 Tecnomed Foundation, University of Milano-Bicocca, Monza, Italy

3 Department of Pathophysiology and Transplantation, University of Milan, Milan, Italy

4 Department of Medical Oncology, IRCCS Fondazione Maugeri, Pavia, Italy

5 Laboratory of Experimental Oncology and Pharmacogenomics, Institutional Oncologic Bio-Bank "Bruno Boerci", IRCCS Fondazione Salvatore Maugeri, Pavia, Italy analysis, is upregulated in highly aggressive MDA-MB231 breast cancer cell line and patient tissues with poor prognosis with respect to good prognosis.

Conclusions Our results suggest a potential role of $\mathrm{Hsa}$ $m i R-567$ as a novel prognostic biomarker for $\mathrm{BC}$ and as regulator of KPNA4.

Keywords MicroRNA/miRNA - Breast cancer .

Prognosis · Biomarker · Proliferation

\section{Introduction}

Breast cancer (BC) is one of the most common cancers worldwide and the most frequent women tumor ( $25 \%$ of all new cases diagnosed in 2015) [14, 25]. Although the current histological and biological indexes (i.e., tubule formation, nuclear polymorphism, mitotic count,...) $[2,10]$ have shown association with clinical outcome of $\mathrm{BC}$ patients, these indexes are limited in their ability to make prognosis for all $\mathrm{BC}$ patients.

With the advent of microarray and the sequencing of the human genome, transcriptomic analysis has significantly improved the knowledge of the biological mechanisms leading to malignant transformation of $\mathrm{BC}$, providing better molecular 'portrait' of $\mathrm{BC}$ and allowing the identification of new BC prognostic and predictive tools.

Several gene profiles have been proposed for the classification of BC [i.e., the 105-gene profile by Perou [24], 70-gene profile in Mammaprint by van 't Veer [29], 21-gene profile in Oncotype by Paik [22], 97 gene profile by Sotiriou [26], 18 gene profile, and 6 gene profile by Ivshina [13]]. Notwithstanding these gene profiles have few genes in common, they have consistently shown that $\mathrm{BC}$ patients can be classified into two groups with different 
prognosis, namely grade 1 (G1)-like and grade 3 (G3)-like groups, representing the two different prognostic groups defined by histological analysis as G1 and G3 [13].

MicroRNAs (miRNAs or miRs) are small, highly conserved, non-coding RNAs that regulate gene expression of target mRNAs. They are widely involved in several physiologic processes, but they are also responsible for pathological conditions, such as carcinogenesis and cancer development [11]. miRNA profiles obtained by 'omics'based technology can classify human cancers even better than mRNA profile $[19,27]$. Moreover, miRNAs are emerging as easy accessible cancer disease biomarkers, as they have been found stably present in human biofluids (i.e., blood, saliva, urine...) $[3,4]$. These features make miRNAs interesting, non-invasive, and cost affordable biomarkers for clinical diagnosis, prognosis, and therapy of BC.

By combining gene expression profile, copy number alterations, and miRNA expression profiles as available from published datasets of $\mathrm{BC}$ human tissue samples, we have recently identified in silico, a 4-miRNA signature ( $\mathrm{Hsa}-\mathrm{miR}$ 567, Hsa-miR-139-5p, Hsa-miR-320d, Hsa-Let-7c) and a 4-target mRNA signature (KPNA4, H2AFV, FOXM1, $D D X 19 A$ ) that are able to accurately classify BC patients into G1-like and G3-like groups, thus improving BC grade definition and confirming the existence of only two prognostic groups [5]. Our signature was the first one, to our knowledge, consisting of miRNAs with these prognostic properties in BC. In particular, it was the first time that Hsa-miR-567 $(m i R-567)$ was found significantly downregulated in BC.

Following our preliminary in silico results, the present study aims at elucidating the role of $m i R-567$ for the prognosis of $\mathrm{BC}$ and as regulator of karyopherin 4 (KPNA4), and to investigate the effects caused by a dysregulation of $m i R-567$. Specific aims of our study are to assess the following, by experimental studies: (1) if the expression level of miR-567 is significantly downregulated in $\mathrm{BC}$ with poor prognosis when compared to $\mathrm{BC}$ with good prognosis (and, consistently, if the expression level of KPNA4 is significantly upregulated), and (2) $m i R-567$ modulation effects on $\mathrm{BC}$ tumor cell proliferation and migration. To reinforce the evidences of a crucial role in $\mathrm{BC}$ of KPNA4 and miR-567, we implemented a classification algorithm and evaluated the performances of proposed biomarkers in the classification of $\mathrm{G} 1$ and $\mathrm{G} 3 \mathrm{BC}$ and in the re-classification of G2, using an independently collected Gene Expression Omnibus (GEO) dataset.

\section{Materials and methods}

\section{BC cell line}

For in vitro studies, we used two human $\mathrm{BC}$ epithelial cell lines: G1-like MCF7 and G3-like MDA-MB-231 cells [12]
(ICLC-Biologic Bank and Cell Factory, Italy). We have chosen MCF7 and MDA-MB-231 cell lines for their ability to form moderately well-differentiated infiltrating ductal carcinomas (grade I) and poorly differentiated adenocarcinoma (grade III), respectively, when xenografted in mouse [https://www.lgcstandards-atcc.org/Products/All/ HTB-26.aspx\#characteristics] [12, 31]. Following the manufacturer's recommendation, we maintained the cell lines within a humidified atmosphere containing $5 \% \mathrm{CO}_{2}$ at $37^{\circ} \mathrm{C}$ in DMEM cell culture medium (Gibco, Life Technologies), with $10 \%$ fetal bovine serum (FBS) (Lonza, Euroclone). Dulbecco Phosphate-Buffered Saline (D-PBS), trypsin, and all the media additives (penicillin, streptomycin, D-glutamate, non-essential amino acids) were obtained by Lonza (Euroclone).

\section{BC human tissue samples}

We used 13 G1 and 13 G3 snap-frozen human BC tissues, diagnosed histopathologically at "Salvatore Maugeri" Foundation, at the Histopathology Service, from 2009 to 2015. G1 and G3 grade definition was established according to the Scarff-Bloom-Richardson (SBR) grading system [1].Tissue samples were immediately stored as frozen aliquots in the "Bruno Boerci" Institutional Oncologic Bio-bank after surgery until further use, according to the guidelines of European Bio-banking and Biomolecular Resource.

\section{BC animals and animal tissue samples}

Fourteen adult female nude mice (athymic nude-Foxn1 Nu/ $\mathrm{Nu}$ ), 7-8 weeks old, were used for the in vivo experiment, and xenografted to model BC.

During in vivo study, mice were maintained on a $12-\mathrm{h}$ light-dark cycle in cages of five animals with water and food ad libitum.

For ex vivo studies, at the end of the experiments, mice were sacrificed by cervical dislocation after sedation and five explanted tumors, from five mice were immediately stored in liquid nitrogen for further use.

\section{RNA isolation and reverse transcription (in vitro and ex vivo studies)}

Total RNA was isolated using TRIzol reagent (Life Technologies) following the manufacturer's recommendations.

Two micrograms of total RNA were reverse transcribed using miRCURY LNA Universal RT microRNA PCR (Exiqon, Euroclone) to obtain poly-A-tailed miRNA cDNA. U6 spike-in RNA was added in the reaction as an internal control for the reverse transcription and real-time PCR (RT-PCR) amplification. 
Two micrograms of total RNA, treated with DNAse I (Euroclone, Italy), were reverse transcribed using oligo dT primers in combination with SuperScript II Reverse Transcriptase (Life Technologies) in order to obtain total cDNA for gene expression analysis, following the manufacturer's protocol.

\section{RT-PCR for analysis of $m i R-567$ and $K P N A 4$ expression levels}

miR-567 and KPNA4 expression levels were analyzed on BC cell lines and human samples. RT-PCR was performed using SybrSelect Master Mix (Applied Biosystem, Life Technologies), in an Eco RT-PCR (Illumina).

Primer sequences for $m i R-567$ expression analysis have been purchased from Exiqon (Euroclone, Italy) (Accession No. MI0003573). The level of expression of $m i R-567$ was normalized on the level of U6 control gene.

Primer sequences for gene expression analysis are: for KPNA4 (ID3840) Fw: 5'- CAGGAGATTCTTCCAGCC CTTTGTGT-3', Rw: 5'- ATTACCATCTGTATTTGTT CATTGCCAGCATC-3'. For ribosomal protein S14 (RPS14) (ID6208) Fw: 5' - GGCAGACCGAGATGAATC CTCA-3', Rw: 5'- CAGGTCCAGGGGTCTTGGTCC-3'. RPS14 was used as internal control for RT-PCR quantification.

The relative expression of miR-567 and KPNA4 was calculated for both G3 and G1 cell lines and human samples with the $2^{\left(-\Delta \Delta \mathrm{C}_{\mathrm{T}}\right)}$ method [17]. Experiments were performed three times in triplicate $(n=9)$. A $t$ test was calculated among G3 and G1 cell lines and human samples.

\section{miR-567 stable transfectant generation in G3-like cell line: $m i R-567$ and $K P N A 4$ analyses}

MDA-MB-231 cells stably overexpressing miR-567 (MDA-miR) or scramble sequence (MDA-Scr) (for the scramble sequence see [18]) were obtained by seeding 50,000 cells in 24-well plate (Euroclone) and transfecting them with $500 \mathrm{ng}$ of pCMV-miR567-GFP (Origene, Nockville, MD) or $100 \mathrm{nM}$ scramble, respectively, in the presence of Lipofectamine 2000 reagent (Invitrogen), following the manufacturer's suggestions. After $48 \mathrm{~h}$, the medium was changed, and G418/Neomycin (Euroclone) $500 \mathrm{ug} / \mathrm{ml}$ was added. The whole population was maintained for 3 weeks in selection (3 passages/week) to obtain a stable transfectant cell line.

After 3 weeks, to assess the efficiency of the stable cell transfection, we evaluated the expression levels of $m i R-567$ and KPNA4 in the whole population of MDA-miR versus MDA-Scr by RT-PCR, using the same protocol described above.
miR-567 stable transfectant generation in $\mathbf{G 3}$ cell line: Kpna4 protein analysis

Total lysates were obtained by lysing $1 \times 10^{6}$ of MDA-ctr cells and MDA-miR cells $30 \mathrm{~min}$ in RIPA lysis buffer (50 mM Tris, pH 7.5, 150 mM NaCl, 1\% NP-40, 2\% SDS, $0.15 \%$ deoxycholic acid, 1 mM EDTA, pH 8. 0) containing complete protease inhibitor (Roche, Monza, Italy), leupeptin and aprotinin inhibitors (Sigma Aldrich). Lysates were spun at $12,000 \times g$ for $30 \mathrm{~min}$ and the supernatants collected. Estimation of protein concentration was performed with $660 \mathrm{~nm}$ Protein assay (Pierce, Thermo Scientific, France) on a Fluostar Omega plate reader (BMG Labtech, Germany).

Twenty micrograms of total proteins were loaded on 8.5 , 10 , or $12 \%$ SDS-PAGE and run for $1.5 \mathrm{~h}$ at $30 \mathrm{~mA} / \mathrm{gel}$. Proteins were transferred onto nitrocellulose membrane (Sigma Aldrich) at $250 \mathrm{~mA}$ for $4 \mathrm{~h}$. Membranes were blocked in TBS-0.1\% Tween-20 containing 5\% skim milk powder (Euroclone, Italy), and incubated with primary antibodies: rabbit polyclonal anti-beta Actin (OriGene, MD, USA), anti-Histone H3 (Origene, MD, USA), or antiTubulin (sc-5286, Santa Cruz Biotechnologies) used 1:000; rabbit polyclonal anti-Kpna4 (Thermo Fisher Scientific, IL, USA) diluted 1:750. After incubation with goat anti-rabbit HRP secondary antibody (sc-2004, Santa Cruz Biotechnologies) or goat anti-mouse HRP (sc-2005, Santa Cruz Biotechnologies) at 1:5000, membranes were developed using the SuperSignal West Pico Chemiluminescent Substrate (ThermoScientific, France) on Amersham Hyperfilm MP (Ge Helthcare, Italy).

To assess the stable transfection, we evaluated the expression levels of Kpna4 protein in MDA-ctr and MDAmiR by Western Blot analysis, normalizing the Kpna4 protein to histone $\mathrm{H} 3$. The blots of both proteins were quantified by ImageJ software (http://imagej.nih.gov/ij/). Experiments were performed two times in triplicate $(n=6)$. A $t$ test was calculated among MDA-miR and MDA-ctr.

\section{Luciferase assay}

To assess if KPNA4 is a direct target of $m i R-567$, pMiRtarget vector, containing the $3^{\prime} \mathrm{UTR}$ of human KPNA4 sequence (Origene, Nashville), was transfected with lipofectamine 3000 (Invitrogen) in MDA-MB-231 cells, according to the manufacturer's suggestion, in combination with $100 \mathrm{nM}$ mimic miR-567 or $100 \mathrm{nM}$ scramble miR sequence. After $24 \mathrm{~h}$, Luciferase activity was measured in GloMax-Multi Detection System (Promega, Madison, WI, USA) and normalized on total protein content. Protein quantification has been performed with Bradford method (Promega). 


\section{In vitro study of tumor cell proliferation}

Tumor cell viability was assessed by following the growth of 50,000 MDA-ctr cells or MDA-miR, seeded in 24-multiwell plate. At time 0,24,48,72 h from seeding, the cells were gently washed withPBS, trypsinized and count with Trypan Blue.

A graphic representation of the results was obtained by plotting the number of the total cells at each time point. Experiments were performed three times in triplicate $(n=9)$. A $t$ test was calculated among MDA-miR and MDA-ctr.

\section{In vitro study of tumor cell migration}

To study directional cell migration, we performed a wound-healing study. The MDA-ctr and MDA-miR cells were seeded at confluence (about 1,50,000/well) in 24-well plate (Euroclone). After $6 \mathrm{~h}$, necessary for adhesion, a scratch was performed in the middle of each well [16]. After washing the cells, new medium was added, and pictures of each well were taken every $24 \mathrm{~h}$. The effect on cell migration was quantified by ImageJ software. Experiments were performed three times in triplicate $(n=9)$. A $t$ test was calculated among MDA-miR and MDA-ctr.

\section{In vivo study of tumor growth}

To engineer cells, a pCCL.PGK.luciferase.WPRE (PLW) lentivector was in vitro used (kindly provided by Dr. S. Rivella). The construct permitted the expression of luciferase reporter gene under control of phosphoglycerate kinase 1 (PGK) promoter.

PLW lentiviral particles were produced by transient transfection into $293 \mathrm{~T}$ cells using calcium phosphate precipitation, as described elsewhere [6]. The transfection medium (IMDM, Euroclone, plus 10\% FBS, Cellgrow) was replaced $16 \mathrm{~h}$ later. The conditioned medium, containing the viral particles, was collected 24 and $48 \mathrm{~h}$ after the medium replacement, filtered through cellulose acetate filter $0.2 \mu \mathrm{m}$, and frozen at $-80{ }^{\circ} \mathrm{C}$ until further use. The vector titer was determined by serial dilution on HeLa cells through Real-Time PCR.

MDA-ctr or MDA-miR cells were plated and infected with PLW particles with a MOI of five in the presence of Polybrene $(8 \mathrm{ug} / \mathrm{ml})$, generating MDA-ctr + Luc (MDALuc) or MDA-miR + Luc (MDA-miR-Luc). Cells were washed $16 \mathrm{~h}$ later in order to remove all viral particles.

Five millions of cells either from MDA-Luc or MDAmiR-Luc were resuspended in $40 \mu \mathrm{l}$ of sterile PBS and injected orthotopically in the fourth mammary gland of the animals. Briefly, mice were anesthetized with Zoletil + Xilor $2 \%$ (40 and $8 \mathrm{mg} / \mathrm{kg}$, respectively) and a 4-mm incision was performed on the skin, in the proximity of the mammary gland. The needle of the syringe was inserted to the mammary gland, and cells were delivered. Following, skin incision was sutured with a $6 / 0$ silk suture thread and mice were followed until complete recovery.

To analyze tumor growth, bioluminescence imaging was performed at different time points. After anesthetization, the 14 mice were injected with $50 \mathrm{mg} / \mathrm{kg}$ of luciferin (Beetle Luciferin Potassium Salt, Promega), and after biodistribution, bioluminescence signal was acquired.

All acquisitions were carried out with IVIS SPECTRUM/CT system (PerkinElmer). Images were analyzed and normalized, using the Living Image Software. Data were expressed as average radiance (photons $/ \mathrm{seconds} / \mathrm{cm}^{2} /$ steradian). The scale and the average radiance used for the images took into account the difference in luciferase activity for the two cell lines.

Dimension of all 14 tumors was measured by caliper on animals at different time points. The tumor volumes were calculated by multiplying the three dimensions of the tumor measured by caliper.

In order to assess if the upregulation of $m i R 567$ was maintained during the in vivo study, five explanted tumors from five xenografted animals, generated by MDA-Luc or MDA-miRLuc, were collected, weighted, and analyzed for RNA, mRNA, and protein content by RT-PCR or Western blot.

\section{Bioinformatic classification approach}

In silico validation analysis using KPNA4 and $m i R-567$ was performed from an independent dataset of GEO database: GSE22220. This dataset contains mRNA and miRNA expression profiles from the same patients: $41 \mathrm{G} 1 \mathrm{BC}, 81$ G2 BC, and $61 \mathrm{G} 3 \mathrm{BC}$ samples.

In order to evaluate the performances of the two proposed biomarkers, we developed a Support Vector Machine (SVM) classification model using the R-package: e1071 [20]. AUC was estimated by cross-validation method (k-fold cross-validation, $k=10$ ).

We optimized inference AUC over a space of given SVM feasible learning parameters: $\operatorname{cost}=10^{(-1: 2)}$, gamma $=$ $\mathrm{c}(0.2,1,2)$; kernel type $=$ RADIAL (see e1071 documentation at [20] (https://CRAN.R-project.org/package=e1071]). This approach allowed us to find the best SVM learning parameters for each data type over the same space of values.

We implemented a Monte Carlo Cross-Validation method. It randomly selected some fractions of GEO data (60\% of original dataset) to form the training set, and then assigned the rest of the points to the testing set $(40 \%$ of original dataset). This process was then repeated multiple times (ten bootstraps), generating randomly new training and test partitions each time. For each bootstrap, we obtained an AUC value. 
In order to avoid problems of unbalanced classes, we randomly selected classes with the same number of BC sample in both the training and testing dataset for each evaluation.

The classifier was tested for the following reasons: (1) to distinguish histological G1 and G3 BC patients using $m i R$ 567 and KPNA4 expression profiles and (2) to re-classify $\mathrm{G} 2$ using miR-567 and KPNA4. For this purpose, the machine learning algorithm based on SVM was used to classify G2 BC patients in G1 or G3 class. The results of testing G2 samples were classified as G1 or G3 (namely G1* and G3*). These classes $\mathrm{G} 1 *$ and $\mathrm{G} 3 *$ were used to train SVM again and to test to the different G1 and G3 BC datasets.

\section{Results}

$m i R-567$ is significantly downregulated and its target KPNA4 is significantly upregulated in G3 versus G1 $\mathrm{BC}$ cell lines

To validate that the expression level of $m i R-567$ is downregulated and KPNA4 mRNA is upregulated in G3 versus G1 BC, we performed a RT-PCR in MDA-MB-231 and MCF7 cell lines. As shown in Fig. 1a, miR-567 expression in MDA-MB-231 is significantly downregulated compared to MCF7 cell line. As shown in Fig. 1b, the relative expression of KPNA4 mRNA is significantly higher in MDA-MB-231 versus MCF7 cell line.

\section{miR-567 is significantly downregulated and KPNA4 is significantly upregulated in G3-like versus G1-like BC human tissues}

To validate that the expression level of $m i R-567$ is downregulated in $\mathrm{G} 3$ versus $\mathrm{G} 1 \mathrm{BC}$ human tissue samples and

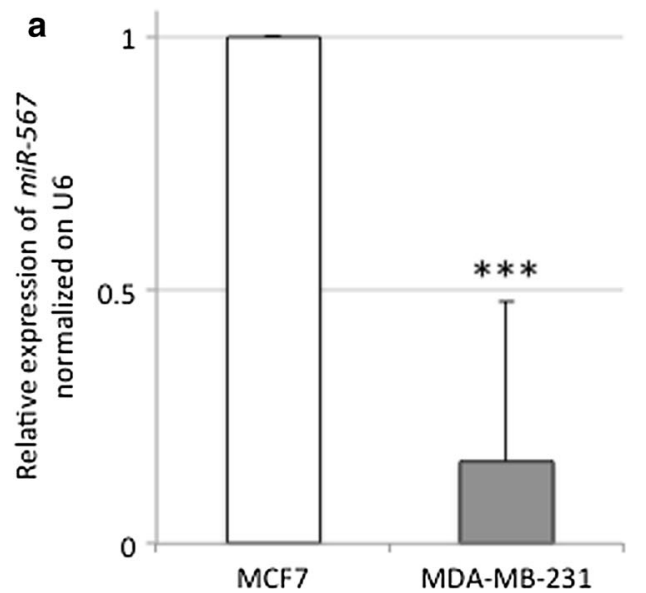

Fig. 1 a RT-PCR relative expression of $m i R-567$ normalized on U6 housekeeping gene, in MCF7 (in white) and MDA-MB-231 (in gray) cell line, respectively. All data are presented as mean \pm SD of experiments for each bar $\left(* * * t\right.$ test $p$ value $\left.=4.5 \times 10^{-5}, n=9\right)$. that expression level of KPNA4 is lower in G1 samples versus G3 samples, we performed a RT-PCR in 13 G3 versus $13 \mathrm{G} 1$ samples. In Fig. 2a, we showed that the expression level of miR-567 is lower in G3 human tissue samples versus G1 samples, with an average of $5.95 \times 10^{-7} \pm 7.40 \times 10^{-7}$ in $\mathrm{G} 1$ and of $6.23 \times 10^{-7} \pm 1.05 \times 10^{-7}$ in G3 samples, respectively. In Fig. 2b, we showed that the expression level of KPNA4 is lower in G1 samples versus G3 samples, with an average of $0.185 \pm 0.218$ in G3 and of $0.093 \pm 0.129$ in G1 samples, respectively.

\section{miR-567 modulation has effects on BC cell} proliferation and migration

Since $m i R-567$ is downregulated in poor prognosis G3 cells, to evaluate the effect of $m i R-567$ upregulation on cell behavior, we generated a stable transfectant, overexpressing miR-567 in MDA-MB-231 cell line. Figure 3a shows that the vector transfection significantly increased $m i R-567$ expression up to $32.15 \pm 8.32$ fold in MDA-miR versus MDA-ctr cells.

To evaluate the effect of $m i R-567$ overexpression on KPNA4 target gene levels, we analyzed both the mRNA levels and the protein levels. As shown in Fig. 3b, miR-567 stable upregulation decreased KPNA4 mRNA expression to $0.57 \pm 0.13$ fold in MDA-miR versus MDA-ctr cells by RT-PCR analysis.

Western blot analysis revealed that Kpna4 protein is also reduced in MDA-miR compared to MDA-ctr cells (Fig. 3c) to $76.34 \pm 18.48$ (ImageJ quantification, Fig. 3d). Luciferase assay confirmed that KPNA4 gene is a direct target of $m i R-567$ (Fig. 3e).

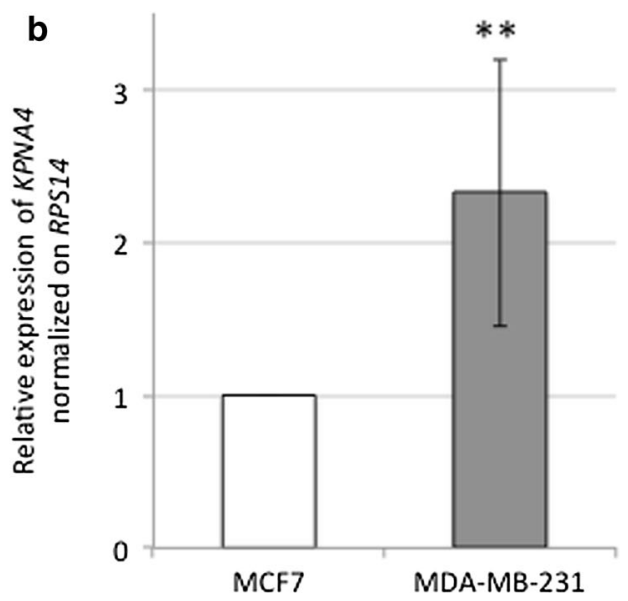

b RT-PCR relative expression of KPNA4 gene normalized on RPS14 housekeeping gene, in MCF7 (in white) and MDA-MB-231 (in gray) cell line respectively. All data are presented as mean \pm SD of experiments for each bar $(* * t$ test $p$ value $=0.0048, n=9$ ) 

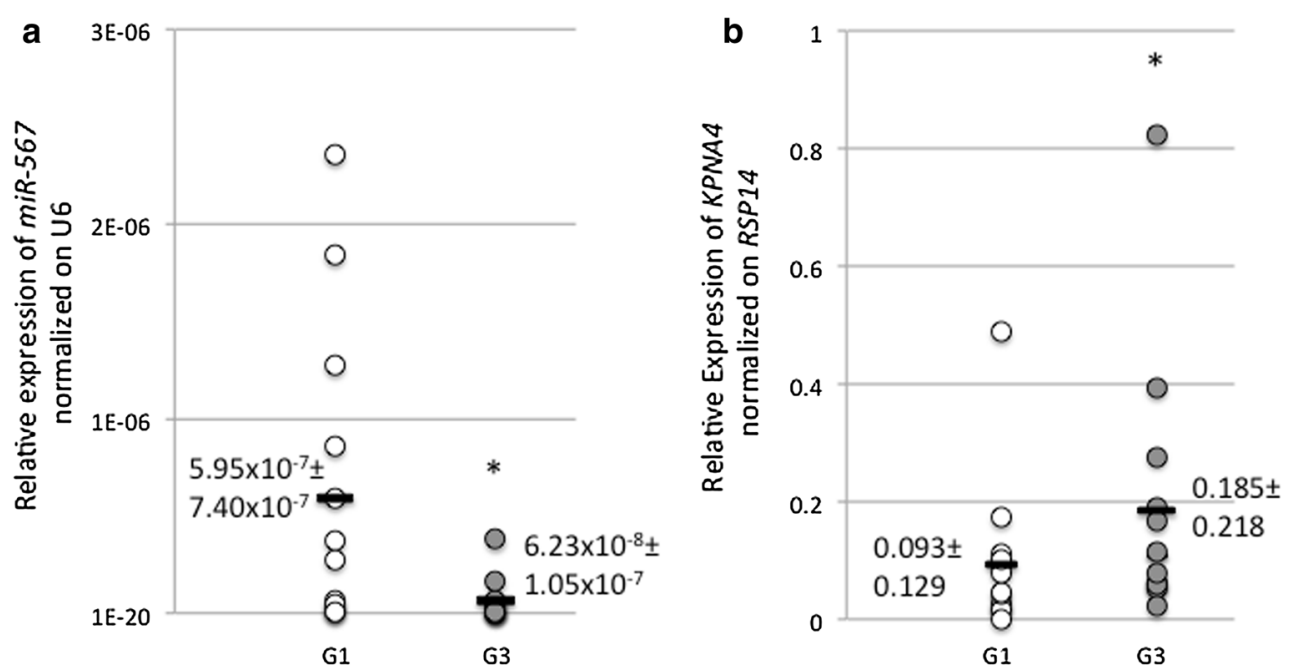

Fig. 2 a Relative expression of $m i R-567$ normalized on U6 in 13 G1 (in white) versus 13 G3 (in gray) BC. All data are presented as mean $\pm \mathrm{SD}$ of experiments for each point. Black bars represent the average of each group. $(* t$ test $p$ value $=0.0223, n=13)$. b Relative

\section{Upregulation of $m i R-567$ decreases tumor cell proliferation}

MDA-miR and the parental MDA-ctr cell lines were tested in growth curve assay (Fig. 4). The overexpression of $m i R$ 567 decreased the proliferation of MDA-miR in respect to MDA-ctr cells (Fig. 4a). The percentage of apoptotic cells was not affected by the overexpression of $m i R-567$ (data not shown).

\section{Upregulation of $m i R-567$ decreases cell migration}

To test the influence of $m i R-567$ overexpression on ability migration of $\mathrm{BC}$ cells, we performed wound-healing assay. MDA-miR showed a lower ability to heal the wound in the MDA-miR cell culture well compared to MDA-Scr cells (Fig. 4b). The difference in the wound area between the two cell lines, quantified by ImageJ, was statistically significant at time 24, 48, and $72 \mathrm{~h}$ (Fig. 4c).

\section{Upregulation of $m i R-567$ decreases in vivo tumor growth}

To test if miR-567 affects the proliferation of BC tumor cells in vivo, we analyzed the behavior of MDA-miR and MDA-ctr implanted in mouse mammary gland. To follow the growth of the tumors, both MDA-miR and MDA-ctr cell lines were engineered in vitro by infection with the PLW construct, producing a detectable signal from luciferase activity. The efficiency of infection was about $90 \%$ (data not shown) and the MDA-miR cells showed a higher level of signal compared to MDA-ctr cells (ratio of MDA-

expression of KPNA4 normalized on RPS14 in 13 G1 (in white) versus 13 G3 (in gray) BC. All data are presented as mean $\pm \mathrm{SD}$ of experiments for each point. Black bars represent the average of each group ( ${ }^{*} t$ test $p$ value $=0.047, n=13$ )

$\mathrm{miR} / \mathrm{MDA}-\mathrm{ctr}=5.7$ fold) - this difference is only due to the differential amount of vector copies integrated in the two cell models (2.125:1 in MDA-miR versus MDA-ctr, respectively) and not to a difference in cell behavior.

As shown in Fig. 5a, a different tumor growing rate was observed by optical imaging: the overexpression of $m i R$ 567 stable construct caused a delay in the growth of the tumors if compared to the growth of MDA-ctr generated tumors (image scale took into account the difference in luciferase constitutive activity of the two cell lines).

The quantification of bioluminescent signal by ROIs showed a very different rate of tumor growth, as demonstrated by the significant $t$ test (Fig. 5b).

This result was confirmed by evaluation of tumor volumes, as recorded by caliper at each time point of signal acquisition, and of tumor weight at the end of experiment, showing a smaller tumor (both in volume and weight) formed by MDAmiR compared to MDA-ctr cells (Fig. 5c-d, respectively).

On the explanted tumors, as expected, miR-567 expression was maintained upregulated in MDA-miR compared to MDA-ctr cells (fold change expression was in mouse 1: $3.03 \pm 1.20$; in mouse $2=2.52 \pm 0.87$; in mouse $3=3.51 \pm 1.65 ;$ in mouse $4=3.22 \pm 0.77$, respectively) (Fig. 6a). One sample (mouse 5) showed an outlier expression of $m i R-567$, with a relative expression of $366.67 \pm 2.34$ fold of increase.

Consistently, on the explanted tumors, KPNA4 expression was found downregulated in MDA-miR compared to MDA-ctr cells (fold change expression of KPNA4 was in mouse 1: $0.01 \pm 0.01$; in mouse $2=0.35 \pm 0.03$; in mouse $3=0.44 \pm 0.14$; in mouse $4=0.33 \pm 0.01$; in mouse $5=0.90 \pm 0.09$, respectively) (Fig. 6b). 

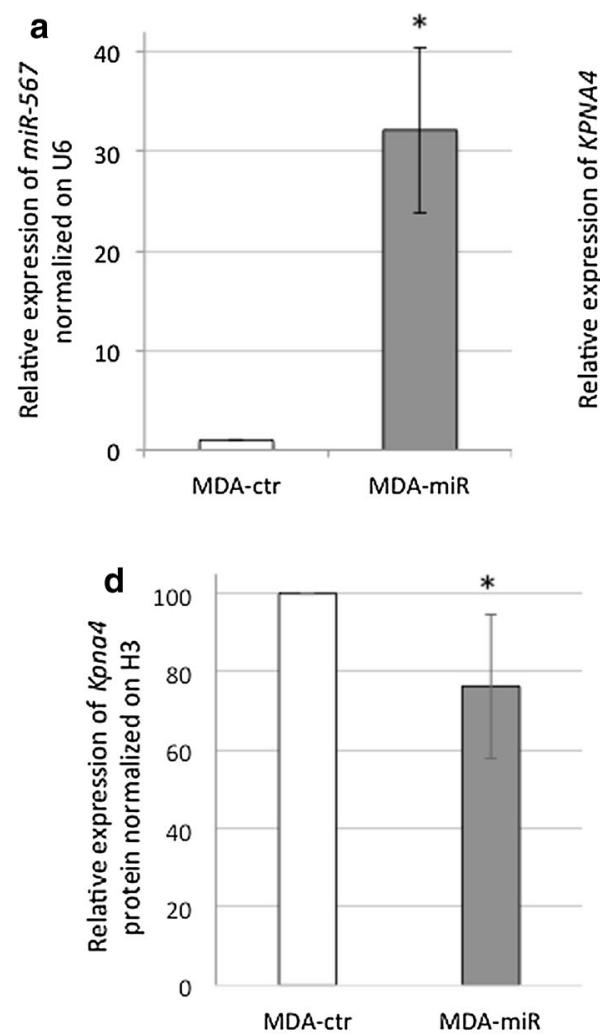

Fig. 3 a Relative expression of $m i R-567$ normalized on $U 6$ in MDActr cells (in white) versus MDA-miR (in gray). All data are presented as mean $\pm \mathrm{SD}$ of experiments for each bar $(* t$ test $p$ value $=0.02$, $n=9$ ). b Relative expression of KPNA4 normalized on RPS14 in MDA-ctr cells (in white) versus MDA-miR (in gray) cell lines, respectively. All data are presented as mean \pm SD of experiments for each bar $(* t$ test $p$ value $=0.012, n=9)$. $\mathbf{c}$ Western Blot of KPNA4 protein and Histone $\mathrm{H} 3$, used as normalizing protein. d Western Blot quantification of KPNA4 protein normalized on Histone H3 in MDActr (in white) and MDA-miR (in gray) cells. All data are presented by mean \pm SD of experiments for each bar $(* t$ test $p$ value $=0.05$, $n=6)$. e Luciferase assay results are depicted in the graph. The

\section{Bioinformatic approach to evaluate the performance of miR-567 and KPNA4 in G1-G3 classification}

Figure 7 shows heat maps of classification performances for G1 versus G3 BC samples, and for the re-classification G2 samples, training on G1*-G3*and testing on G1-G3 for each bootstrap. Mean AUC values showed a good performance of 0.78 [(CI 95\%) 0.71-0.84] and 0.76 [(CI 95\%) 0.74-0.77], respectively. The best performance in G1 versus G3 was obtained in the seventh bootstrap getting an AUC value 0.95. In the re-classification the best performance was 0.8 .

\section{Discussion}

In this study, we explored the role of miR-567 in BC. Our study was based on the results coming from three $\mathrm{BC}$ experimental models: cellular model, mouse model, and human samples. c

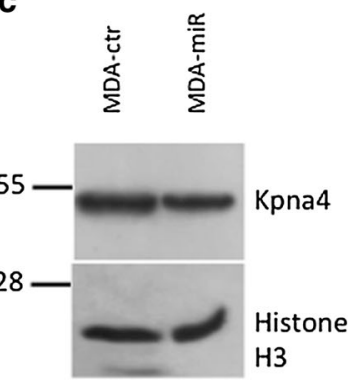

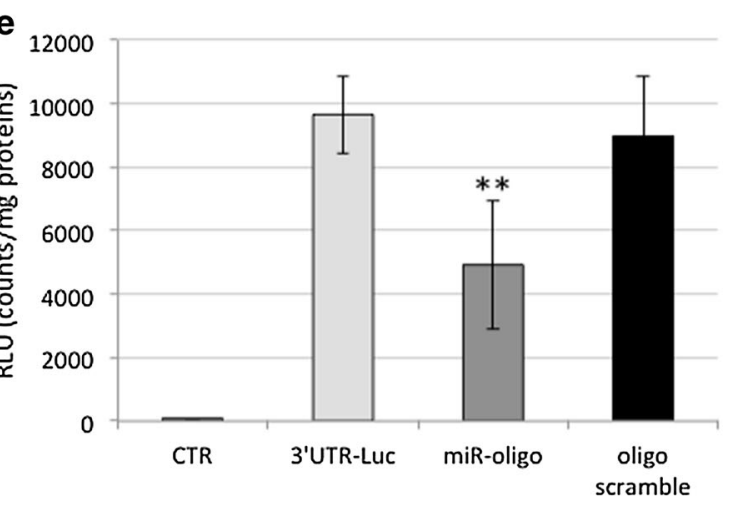

relative luminescence activity (RLU) has been normalized on total protein content for each sample. CTR: untreated MDA-MB-231; $3^{\prime}$ UTR: MDA-MB-231 transfected with pMirTarget vector containing 3'UTR of hKPNA4 sequence; 3'UTR + miRNA: MDA-MB-231 transfected with pMirTarget vector containing $3^{\prime} \mathrm{UTR}$ of hKPNA4 sequence, in combination with $100 \mathrm{nM}$ mimic miR-567 oligonucleotide; $\quad 3^{\prime}$ UTR + scramble: MDA-MB-231 transfected with pMirTarget vector containing $3^{\prime} \mathrm{UTR}$ of hKPNA4 sequence, in combination with $100 \mathrm{nM}$ scramble oligonucleotide. All data are presented as mean $\pm \mathrm{SD}$ of experiments for each point $(* * t$ test $p$ value $=0.0013, n=3$ )

We investigated the role of $m i R-567$ in G3 versus G1 $\mathrm{BC}$ cell lines, and $m i R-567$ was found clearly differentially expressed (Fig. 1a). To better clarify the role that the downregulation of $m i R-567$ has in higher-grade tumor behavior and the genes that are potentially target of $m i R$ 567 regulation, we overexpressed $m i R-567$ construct in MDA-MB-231 cells (Fig. 3a-d). miR-567 upregulation has a drastic effect on cell growth, as revealed by the decrease in cell proliferation (Fig. 4a). In addition, its upregulation strongly affects the migration capability of the cells, as revealed by wound-healing assay (Fig. 4b-c).

In our previous publication [5] by in silico analyses, we had suggested KPNA4 as a possible target of miR-567. Several members of the KPN family are activated in solid tumors (i.e., [9]) and in particular in BC [21]. Kpna4, a nuclear protein with a role in nucleocytoplasmic trafficking, could be involved in signal-transduction pathways and 
a

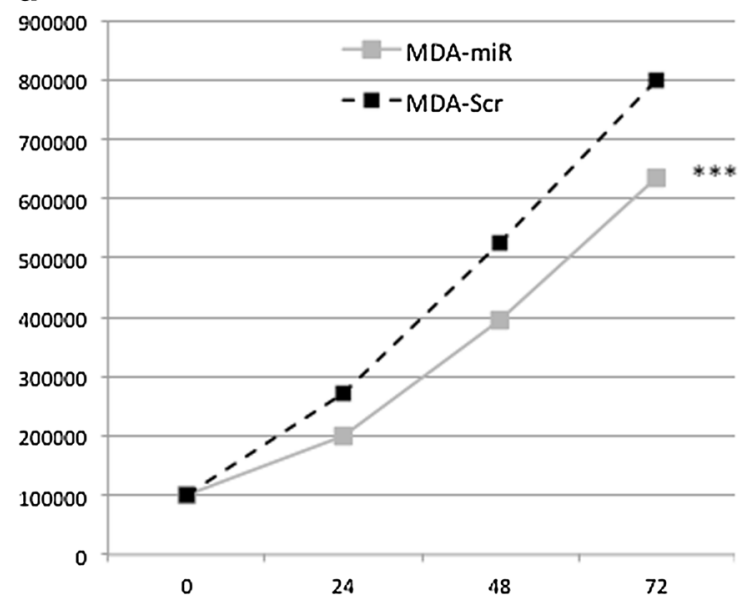

c

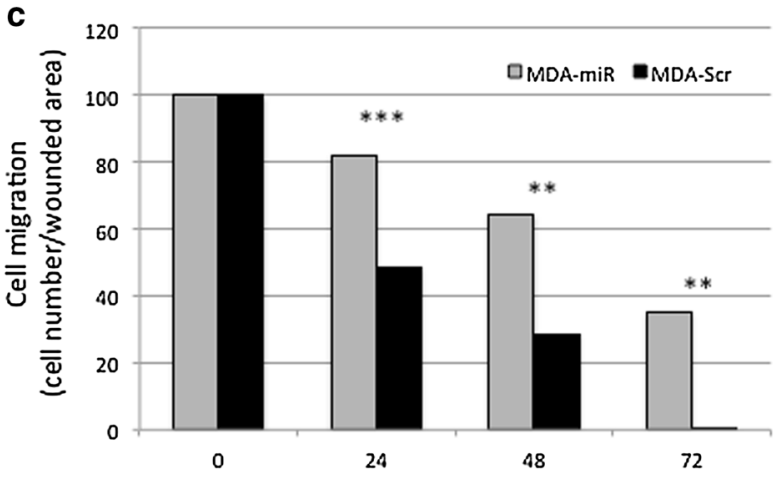

b

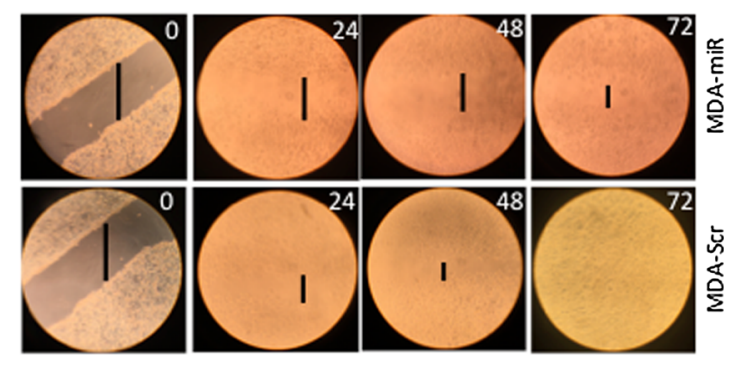

Fig. 4 a Growth curve of MDA-Scr (dashed black line) and MDA$\mathrm{miR}$ (solid gray line) cell lines. All data are presented as mean $\pm \mathrm{SD}$ of experiments for each point at each time point (***t test $p$ value $\left.=1.28 \times 10^{-7}, n=27\right)$. b Behavior of MDA-Scr and MDA-miR cells in wound-healing test. $\mathbf{c}$ ImageJ quantification of the effect of the miR-567 overexpression in MDA-miR compared to MDA-Scr cells, on wound-healing assay. All data are presented as mean $\pm \mathrm{SD}$ of experiments for each bar at each time point (** $p$ value $<0.01,{ }^{* * *} t$ test $p$ value $<0.001, n=3$, respectively)

MDA-miR-generated tumors showed a lower growth rate that those obtained by MDA-ctr cells (Fig. 5). These data confirm that miR-567 is also able in vivo to control the growth rate of highly aggressive BC cells.

The clinical significance of $m i R-567$ in $\mathrm{BC}$ has not been evaluated yet. In the present study, miR-567 downregulation has been detected in poor prognosis G3 human samples compared to better prognosis G1 human samples, suggesting a correlation between $m i R-567$ expression and prognosis (Fig. 2). The same results have been obtained for KPNA4 expression level analysis in G3 versus G1 BC samples (Fig. 2). Also, in silico analysis showed the good performances of $m i R-567$ and KPNA4 to distinguish G1 and G3, and in the re-classification of G2, using an independent dataset (Fig. 7).

No publication associates $m i R-567$ expression levels to $\mathrm{BC}$, although two papers showed, in silico, a correlation between miR-567 expression and colon cancer (CRC) $[8,30]$. In CRC, $m i R-567$ has been proposed as a controller 


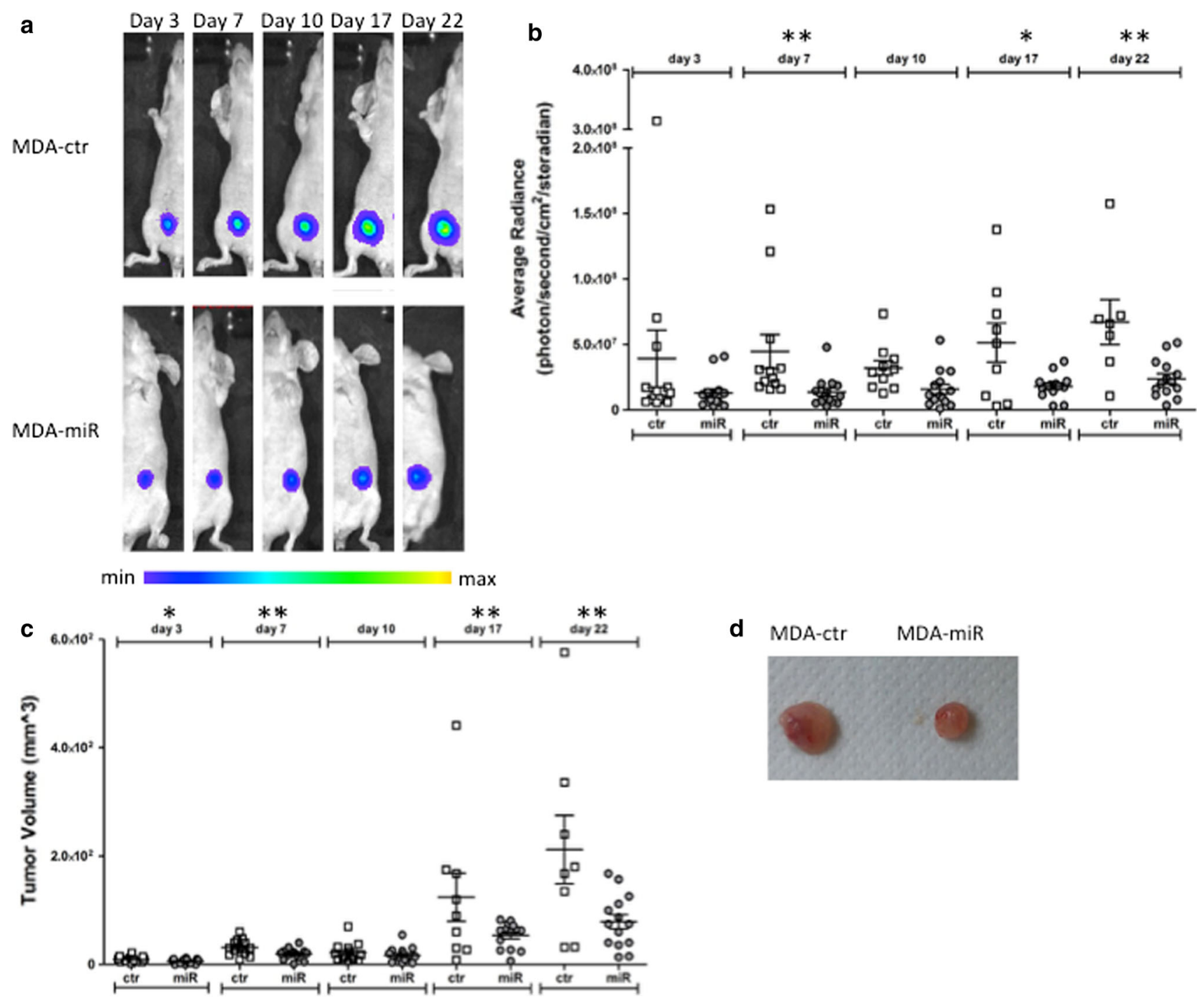

Fig. 5 a Bioluminescence imaging of a representative mouse injected with MDA-ctr (control) cells or with MDA-miR cells. All the images are scaled with the same color bar. b Graphical representation of average of Luciferase activity acquired for each of the tumor formed by MDA-ctr (ctr) and MDA-miR (miR) cell lines, respectively, at different time points (day 3, 7, 10, 17, 22). Data are presented as average radiance (photons $/ \mathrm{s} / \mathrm{cm}^{2} /$ steradian). All the bars are mean \pm SEM of experiments for each cell line at each time point $(* * t$ test $p$ value day $7=0.008 ; * t$ test $p$ value day $17=0.015 ; * *$

of SMAD4 pathway. SMAD4, a major component of the transforming growth factor $\beta$ (TGF- $\beta$ ) signaling pathway, has been found activated in metastatic BC [32]. TGF- $\beta$ pathway is crucial for the invasion and migration of $\mathrm{BC}$ cell in surrounding tissue [23], and it is upregulated in $81 \%$ of the patients with aggressive cancer $[7,15]$. It is possible that miR-567 is modulated by TGF- $\beta$ pathway also in BC and, in turn, regulates some components of this pathway, as SMAD family members. $t$ test $p$ value day $22=0.007, n=14)$. $\mathbf{c}$ Graphical representation of average of volume for each of the tumor formed by MDA-ctr (ctr) and MDA-miR (miR) cell lines, respectively, at each time point (day 37 , $10,17,22)$. All the bars are mean \pm SEM of experiments for each cell line at each time point $(* t$ test $p$ value day $3=0.025 ; * * t$ test $p$ value day $7=0.010 ; * * t$ test $p$ value day $17=0.006 ; * * t$ test $p$ value day $22=0.002, n=14)$. d Representative image of the difference in the volume of explanted tumors, generated by MDA-ctr (left) and MDA-miR (right) cells, respectively

The higher expression of TGF- $\beta$ is able to modulate a component of KPN karyopherin family, namely KPNA2, involved in the control of keratinocyte proliferation and differentiation [29]. We can hypothesize that in a normal cell, low levels of TGF- $\beta$ allow the expression of $m i R-567$, which in turn decrease KPN family members, regulating the cell growth. In highly aggressive $\mathrm{BC}, \mathrm{TGF}-\beta$ is increased, causing possibly the strict decrease in $m i R-567$ expression and consequently the upregulation of KPNA4, 


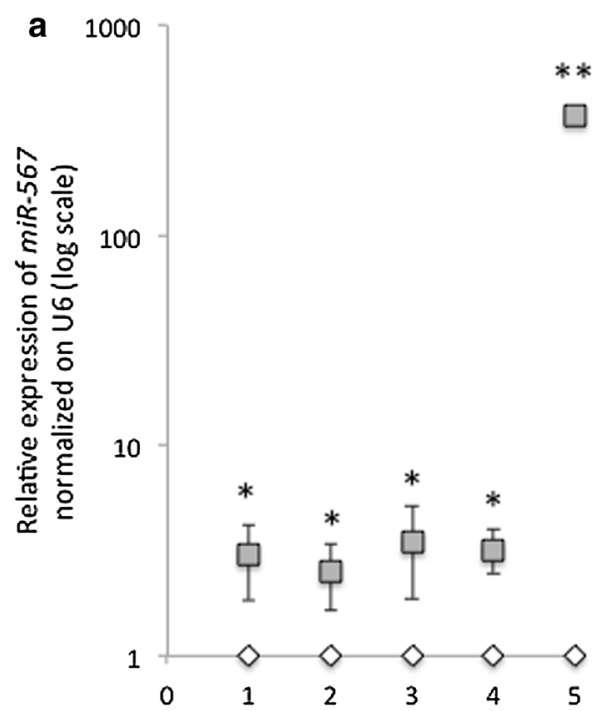

Fig. 6 a Relative expression (log scale) of $m i R-567$ normalized on $\mathrm{U} 6$ on five explanted tumors formed by MDA-miR + luc (in gray) or MDA-ctr + luc (in white) cells, respectively. All data are presented as mean $\pm \mathrm{SD}$ of experiments for each tumor (1-5) ( $t$ test ${ }^{*} p$ value $<0.05$; ** $p$ value $<0.01, n=9$ ). b) Relative expression

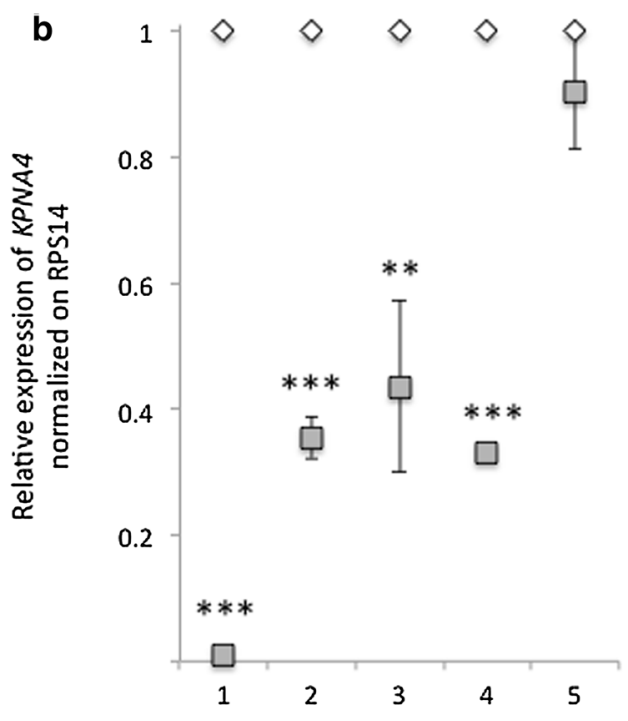

of KPNA4 normalized on RPS14 on five explanted tumors (1-5) formed by MDA-miR + luc (in gray) or MDA-ctr + luc (in white) cells, respectively. All data are presented as mean \pm SD of experiments for each tumor ( $t$ test $* * p$ value $<0.05$; *** $p$ value $<0.001$, $n=9$ )
Fig. 7 Heat maps depicting the predictive performance of KPNA4 and $m i R-567$

classification for G1 versus G3 and for G1 versus $\mathrm{G} 3$, training on $\mathrm{G} 1 *-\mathrm{G} 3 *$, and testing on G1-G3 for each bootstrap b
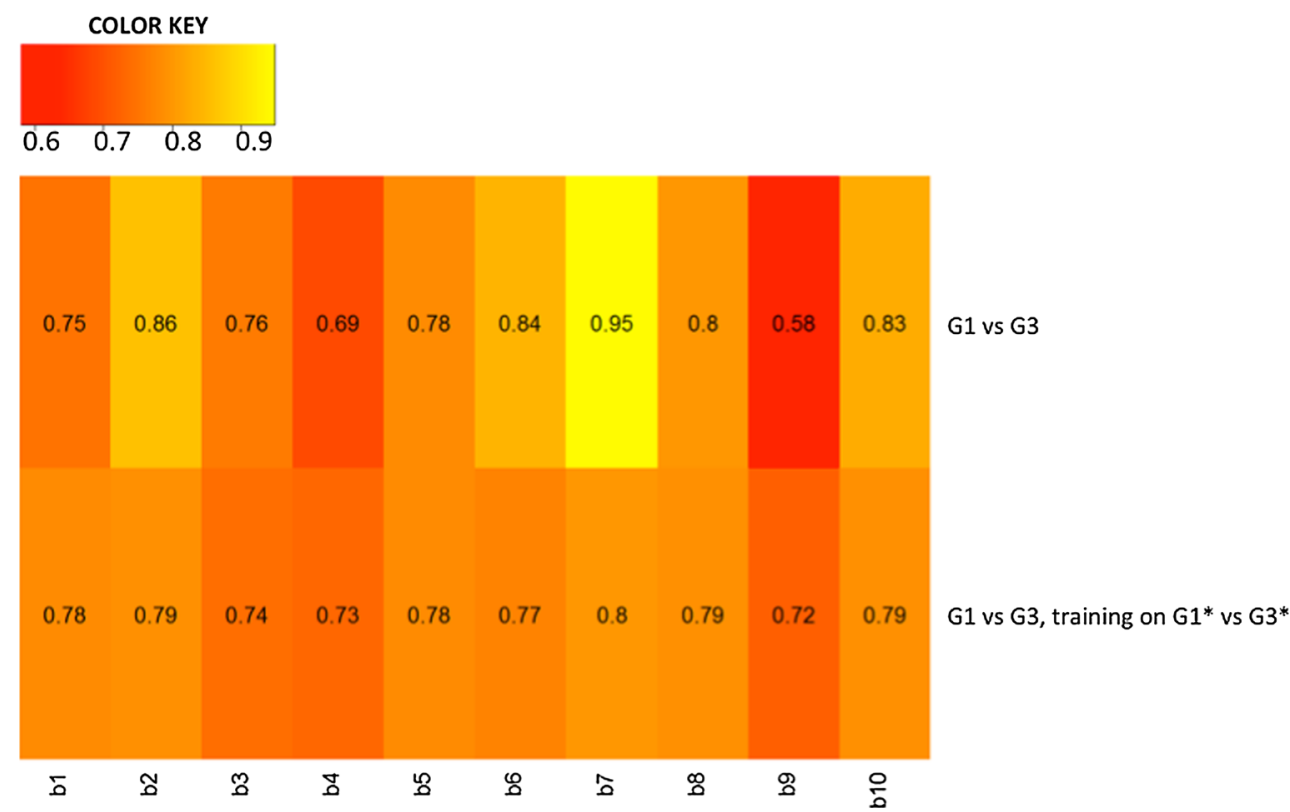

increasing cell proliferation. This hypothesis is depicted in Fig. 8. However, further experiments are needed to confirm it.

In this study, we could propose $m i R-567$ as a therapeutic tool: the use of miR-567 mimic oligonucleotide could be effective in modulating $\mathrm{BC}$ growth through targeting of KPN family members. The balance of $m i R-567$ expression by mimic oligonucleotide administration could become a possible therapeutic strategy for poor prognosis BC.
Several attempts have been made to use miRNAs as possible therapeutic tools [3], but further efforts are needed to achieve this purpose.

In conclusion, we found a high increase of $m i R-567$ in low-risk G1 versus G3 cell lines and even in the corresponding explanted tumors from xenografted mice. A significant difference has been also found between G1 and G3 human BC tissue samples. Our findings suggest that miR567 could act as an oncogene, as its downregulation 


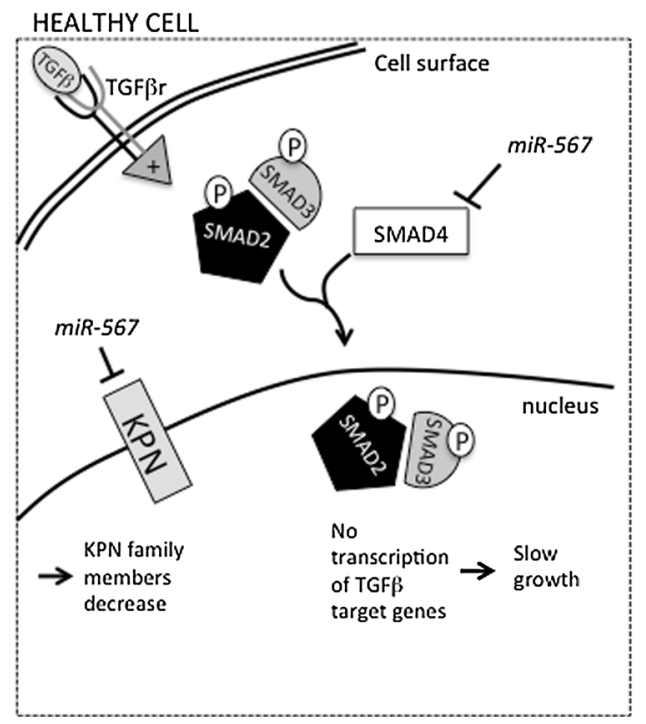

Fig. 8 Hypothesis of $m i R-567$ modulation in healthy and BC cells. When miR-567 is expressed, TGF- $\beta$, bound to its receptor (two subunits), activates the pathway that leads to the phosphorylation of downstream effectors (i.e., SMAD2 and 3) and to the downregulation of KPN family member. The last effect could be mediated by $m i R$ 567 activity. miR-567 could possibly also modulate SMAD4 effector,

decreases cell proliferation in poor prognosis $\mathrm{BC}$, increasing expression level of KPNA4. miR-567 might serve as a novel biomarker in the prognosis of $\mathrm{BC}$ and could be a regulator of KPNA4 expression.

Funding This work was supported partially by Interomics Flagship Project (url: http://www.interomics.eu/it/home) of the National Research Council (Codice Unico di Progetto, CUP Grant B91J12000190001), and by the Project Grant SysBioNet, Italian Roadmap Research Infrastructures.

\section{Compliance with ethical standards}

Conflict of interest The authors declare that they have no conflict of interest.

Ethical approval All procedures performed in studies involving human participants were in accordance with the Ethical standards of the Institutional and/or National Research Comitee. For ex vivo studies on human tissue samples, the experiments were carried out in compliance with the institutional guidelines for the care and use of experimental human samples, which have been approved by the Ethics Committee of the "Salvatore Maugeri" Foundation (Pavia, Italy). Patient consent was obtained. For in vivo and ex vivo studies on animals, the study was approved by the Italian Ministry of Health for the animal facility located at the Department of Pathophysiology and Transplantation, University of Milan (authorization protocol number 960/2015-PR). Animals were managed according to the principles of the "Guide for the Care and Use of Laboratory Animals" [28], in accordance with the Italian national law (Legislative Decree. 26/2014) and the recommendations of the European Community $(63 / 2010 / \mathrm{CEE})$.

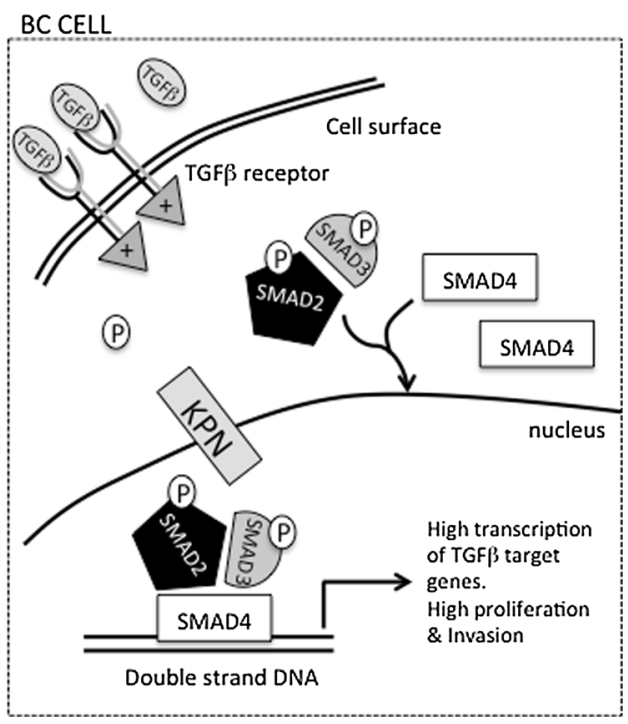

needed for the transcriptional activation of TGF- $\beta$ target genes. In aggressive BC cell, TGF- $\beta$ is upregulated, and the higher activation of its pathway leads to transcription of genes involved in the proliferation control and invasion. The reduction of miR-567 observed in aggressive BC could lead to increase of KPN family members, facilitating the growth of the tumor. TGF $\beta r=$ TGF $\beta$ receptor

Open Access This article is distributed under the terms of the Creative Commons Attribution 4.0 International License (http://crea tivecommons.org/licenses/by/4.0/), which permits unrestricted use, distribution, and reproduction in any medium, provided you give appropriate credit to the original author(s) and the source, provide a link to the Creative Commons license, and indicate if changes were made.

\section{References}

1. Amat S, Penault-Llorca F, Cure H, Le Bouedec G, Achard JL, Van Praagh I, Feillel V, Mouret-Reynier MA, Dauplat J, Chollet P (2002) Scarff-Bloom-Richardson (SBR) grading: a pleiotropic marker of chemosensitivity in invasive ductal breast carcinomas treated by neoadjuvant chemotherapy. Int J Oncol 20:791-796

2. Baxter E, Gondara L, Lohrisch C, Chia S, Gelmon K, Hayes M, Davidson A, Tyldesley S (2015) Using proliferative markers and Oncotype DX in therapeutic decision-making for breast cancer: the B.C. experience. Curr Oncol 22:192-198. doi:10.3747/co.22. 2284

3. Bertoli G, Cava C, Castiglioni I (2015) MicroRNAs: new biomarkers for diagnosis, prognosis, therapy prediction and therapeutic tools for breast cancer. Theranostics 5:1122-1143. doi: 10.7150/thno. 11543

4. Bertoli G, Cava C, Castiglioni I (2016) MicroRNAs as biomarkers for diagnosis, prognosis and theranostics in prostate cancer. Int J Mol Sci. doi:10.3390/ijms17030421

5. Cava C, Bertoli G, Ripamonti M, Mauri G, Zoppis I, Della Rosa PA, Gilardi MC, Castiglioni I (2014) Integration of mRNA expression profile, copy number alterations, and microRNA expression levels in breast cancer to improve grade definition. PLoS ONE 9:e97681. doi:10.1371/journal.pone.0097681

6. Chen C, Okayama H (1987) High-efficiency transformation of mammalian cells by plasmid DNA. Mol Cell Biol 7:2745-2752 
7. Dave H, Shah M, Trivedi S, Shukla S (2012) Prognostic utility of circulating transforming growth factor beta 1 in breast cancer patients. Int J Biol Mark 27:53-59. doi:10.5301/JBM.2011.8736

8. El-Murr N, Abidi Z, Wanherdrick K, Svrcek M, Gaub MP, Flejou JF, Hamelin R, Duval A, Lesuffleur T (2012) MiRNA genes constitute new targets for microsatellite instability in colorectal cancer. PLoS ONE 7:e31862. doi:10.1371/journal.pone.0031862

9. Grupp K, Habermann M, Sirma H, Simon R, Steurer S, HubeMagg C, Prien K, Burkhardt L, Jedrzejewska K, Salomon G, Heinzer H, Wilczak W, Kluth M, Izbicki JR, Sauter G, Minner S, Schlomm T, Tsourlakis MC (2014) High nuclear karyopherin alpha 2 expression is a strong and independent predictor of biochemical recurrence in prostate cancer patients treated by radical prostatectomy. Mod Pathol 27:96-106. doi:10.1038/modpathol. 2013.127

10. Harris L, Fritsche H, Mennel R, Norton L, Ravdin P, Taube S, Somerfield MR, Hayes DF, Bast RC Jr, American Society of Clinical O (2007) American Society of Clinical Oncology 2007 update of recommendations for the use of tumor markers in breast cancer. J clin oncol 25:5287-5312. doi:10.1200/JCO.2007.14.2364

11. Hayes J, Peruzzi PP, Lawler S (2014) MicroRNAs in cancer: biomarkers, functions and therapy. Trends Mol Med 20:460-469. doi:10.1016/j.molmed.2014.06.005

12. Holliday DL, Speirs V (2011) Choosing the right cell line for breast cancer research. Breast Cancer Res 13:215. doi:10.1186/ bcr2889

13. Ivshina AV, George J, Senko O, Mow B, Putti TC, Smeds J, Lindahl T, Pawitan Y, Hall P, Nordgren H, Wong JE, Liu ET, Bergh J, Kuznetsov VA, Miller LD (2006) Genetic reclassification of histologic grade delineates new clinical subtypes of breast cancer. Cancer Res 66:10292-10301. doi:10.1158/0008-5472. CAN-05-4414

14. Kohler BA, Sherman RL, Howlader N, Jemal A, Ryerson AB, Henry KA, Boscoe FP, Cronin KA, Lake A, Noone AM, Henley SJ, Eheman CR, Anderson RN, Penberthy L (2015) Annual Report to the Nation on the Status of Cancer, 1975-2011, featuring incidence of breast cancer subtypes by race/ethnicity, poverty, and state. J Natl Cancer Inst. doi:10.1093/jnci/djv048

15. Kong FM, Anscher MS, Murase T, Abbott BD, Iglehart JD, Jirtle RL (1995) Elevated plasma transforming growth factor-beta 1 levels in breast cancer patients decrease after surgical removal of the tumor. Ann Surg 222:155-162

16. Liang CC, Park AY, Guan JL (2007) In vitro scratch assay: a convenient and inexpensive method for analysis of cell migration in vitro. Nat Protoc 2:329-333. doi:10.1038/nprot.2007.30

17. Livak KJ, Schmittgen TD (2001) Analysis of relative gene expression data using real-time quantitative PCR and the 2(-Delta Delta C(T)) Method. Methods 25:402-408. doi:10.1006/meth. 2001.1262

18. Lo Dico A, Costa V, Martelli C, Diceglie C, Rajata F, Rizzo A, Mancone C, Tripodi M, Ottobrini L, Alessandro R, Conigliaro A (2016) MiR675-5p acts on HIF-1alpha to sustain hypoxic responses: a new therapeutic strategy for glioma. Theranostics 6:1105-1118. doi:10.7150/thno.14700

19. Lu J, Getz G, Miska EA, Alvarez-Saavedra E, Lamb J, Peck D, Sweet-Cordero A, Ebert BL, Mak RH, Ferrando AA, Downing JR, Jacks T, Horvitz HR, Golub TR (2005) MicroRNA expression profiles classify human cancers. Nature 435:834-838. doi:10.1038/nature03702

20. Meyer D, Dimitriadou E, Hornik K, Weingessel A and Leisch F (2015) Misc functions of the department of statistics, Probability Theory Group In: e1071. TU Wien. R package version 1.6-7

21. Noetzel E, Rose M, Bornemann J, Gajewski M, Knuchel R, Dahl E (2012) Nuclear transport receptor karyopherin-alpha 2 promotes malignant breast cancer phenotypes in vitro. Oncogene 31:2101-2114. doi:10.1038/onc.2011.403
22. Paik S, Shak S, Tang G, Kim C, Baker J, Cronin M, Baehner FL, Walker MG, Watson D, Park T, Hiller W, Fisher ER, Wickerham DL, Bryant J, Wolmark N (2004) A multigene assay to predict recurrence of tamoxifen-treated, node-negative breast cancer. N Eng J Med 351:2817-2826. doi:10.1056/NEJMoa041588

23. Pang MF, Georgoudaki AM, Lambut L, Johansson J, Tabor V, Hagikura K, Jin Y, Jansson M, Alexander JS, Nelson CM, Jakobsson L, Betsholtz C, Sund M, Karlsson MC, Fuxe J (2016) TGF-beta1-induced EMT promotes targeted migration of breast cancer cells through the lymphatic system by the activation of CCR7/CCL21-mediated chemotaxis. Oncogene 35:748-760. doi:10.1038/onc.2015.133

24. Perou CM, Sorlie T, Eisen MB, van de Rijn M, Jeffrey SS, Rees CA, Pollack JR, Ross DT, Johnsen H, Akslen LA, Fluge O, Pergamenschikov A, Williams C, Zhu SX, Lonning PE, Borresen-Dale AL, Brown PO, Botstein D (2000) Molecular portraits of human breast tumours. Nature 406:747-752. doi:10.1038/ 35021093

25. Society AC (2016) Cancer facts \& figures 2016. American Cancer Society, Atlanta

26. Sotiriou C, Wirapati P, Loi S, Harris A, Fox S, Smeds J, Nordgren H, Farmer P, Praz V, Haibe-Kains B, Desmedt C, Larsimont D, Cardoso F, Peterse H, Nuyten D, Buyse M, Van de Vijver MJ, Bergh J, Piccart M, Delorenzi M (2006) Gene expression profiling in breast cancer: understanding the molecular basis of histologic grade to improve prognosis. J Natl Cancer Inst 98:262-272. doi:10.1093/jnci/djj052

27. Sun EH, Zhou Q, Liu KS, Wei W, Wang CM, Liu XF, Lu C, Ma DY (2014) Screening miRNAs related to different subtypes of breast cancer with miRNAs microarray. Eur Rev Med Pharmacol Sci 18:2783-2788

28. The NRCUCftUotGf, Animals CaUoL (2011) Guide for the care and use of laboratory animals, 8th edn. National Academies Press (US), Washington (DC)

29. Umegaki N, Tamai K, Nakano H, Moritsugu R, Yamazaki T, Hanada K, Katayama I, Kaneda Y (2007) Differential regulation of karyopherin alpha 2 expression by TGF-beta1 and IFN-gamma in normal human epidermal keratinocytes: evident contribution of KPNA2 for nuclear translocation of IRF-1. J Invest Dermatol 127:1456-1464. doi:10.1038/sj.jid.5700716

30. van 't Veer LJ, Dai H, van de Vijver MJ, He YD, Hart AA, Mao M, Peterse HL, van der Kooy K, Marton MJ, Witteveen AT, Schreiber GJ, Kerkhoven RM, Roberts C, Linsley PS, Bernards R, Friend SH (2002) Gene expression profiling predicts clinical outcome of breast cancer. Nature 415:530-536. doi:10.1038/ 415530a

31. Wang F, Wong SC, Chan LW, Cho WC, Yip SP, Yung BY (2014) Multiple regression analysis of mRNA-miRNA associations in colorectal cancer pathway. Biomed Res Int 2014:676724. doi:10.1155/2014/676724

32. Xie D, Miller CW, O’Kelly J, Nakachi K, Sakashita A, Said JW, Gornbein J, Koeffler HP (2001) Breast cancer. Cyr61 is overexpressed, estrogen-inducible, and associated with more advanced disease. J Biol Chem 276:14187-14194. doi:10.1074/jbc. M009755200

33. Xue J, Lin X, Chiu WT, Chen YH, Yu G, Liu M, Feng XH, Sawaya R, Medema RH, Hung MC, Huang S (2014) Sustained activation of SMAD3/SMAD4 by FOXM1 promotes TGF-betadependent cancer metastasis. J Clin Invest 124:564-579. doi:10. 1172/JCI71104

34. Zannini L, Lecis D, Lisanti S, Benetti R, Buscemi G, Schneider C, Delia D (2003) Karyopherin-alpha2 protein interacts with Chk2 and contributes to its nuclear import. J Biol Chem 278:42346-42351. doi:10.1074/jbc.M303304200 